\title{
Immune cartography of macrophage activation syndrome in the COVID-19 era
}

\author{
Dennis McGonagle ${ }^{1,2 \bowtie}$, Athimalaipet V. Ramanan ${ }^{3}$ and Charlie Bridgewood ${ }^{1}$
}

Abstract | A hyperinflammatory 'cytokine storm' state termed macrophage activation syndrome (MAS), culminating from a complex interplay of genetics, immunodeficiency, infectious triggers and dominant innate immune effector responses, can develop across disparate entities including systemic juvenile idiopathic arthritis (sJIA) and its counterpart adult-onset Still disease (AOSD), connective tissue diseases, sepsis, infection, cancers and cancer immunotherapy. Classifying MAS using the immunological disease continuum model, with strict boundaries that define the limits of innate and adaptive immunity, at one boundary is MAS with loss of immune function, as occurs in the 'perforinopathies' and some cases of sJIA-AOSD. Conversely, at the other boundary, immune hypersensitivity with gain of immune function in MHC class II-associated sJIA-AOSD and with chimeric antigen receptor (CAR) T cell therapy also triggers MAS. This provides a benchmark for evaluating severe inflammation in some patients with COVID-19 pneumonia, which cripples primary type I interferon immunity and usually culminates in a lung-centric 'second wave' cytokine-driven alveolitis with associated immunothrombosis; this phenomenon is generally distinct from MAS but can share features with the proposed 'loss of immune function' MAS variant. This loss and gain of function MAS model offers immune cartography for a novel mechanistic classification of MAS with therapeutic implications.

${ }^{1}$ Leeds Institute of Rheumatic and Musculoskeletal Medicine, University of Leeds, Leeds, UK.

${ }^{2}$ National Institute for Health Research (NIHR), Leeds Biomedical Research Centre (BRC), Leeds Teaching Hospitals, Leeds, UK.

${ }^{3}$ University Hospitals Bristol NHS Foundation Trust \& Translational Health Sciences, University of Bristol, Bristol, UK.

凶e-mail:d.g.mcgonagle@ leeds.ac.uk

https://doi.org/10.1038 s41584-020-00571-1
The term 'cytokine storm' entered the popular lexicon with previous influenza virus pandemics but has become even more mainstream because of the severe inflammation that can accompany SARS-CoV-2 infection ${ }^{1}$. Despite the availability of contemporary immunological techniques that can measure cytokines down to the femtograms per millilitre range, there is no definition of what actually constitutes a cytokine storm ${ }^{1}$; however, in the broadest sense, the term refers to high cytokine concentrations in specific clinical contexts. Many scenarios, including infection, aberrant immunity, cancer and cancer immunotherapy, are associated with cytokine storm, also known as cytokine release syndrome (CRS) or hypercytokinaemia. Although the cytokine storm concept is poorly defined, it can certainly evolve, sometimes rapidly, into different clinico-pathological entities. One of these entities is macrophage activation syndrome (MAS), which is typified by fever, variable hepatosplenomegaly, elevations in CRP concentration, hyperferritinaemia, haemophagocytosis, cytopenias (including pancytopenia), and coagulopathy linked to liver dysfunction and to disseminated intravascular coagulation (DIC) (reviewed thoroughly elsewhere $^{2-4}$ ).
The MAS terminology originally emerged in rheumatology in relation to systemic juvenile idiopathic arthritis (sJIA) and adult-onset Still disease (AOSD), with both considered to be part of the same disease continuum, in which context MAS was also termed secondary haemophagocytic lymphohistiocytosis (HLH; also known as reactive or acquired HLH) because of the striking overlap with primary HLH (also known as familial HLH $)^{5}$, which was originally defined as a paediatric monogenic immunodeficiency state with hyperinflammation ${ }^{6}$. It is clear that MAS has a complex relationship with infectious disease, but this relationship is not well defined. Many viral diseases, including the contemporary COVID-19 caused by SARS-CoV-2, have been linked to a MAS-type phenotype with hyperferritinaemia and DIC, but the incidence of this classical MAS picture in COVID-19 awaits fuller description ${ }^{7}$. Indeed, the COVID-19 pandemic has brought the issue of cytokine storm and MAS to a wider medical and immunology audience. However, as outlined below, therapies that can be curative in MAS are not especially effective in severe COVID-19, which has not been adequately explained. In this article, we propose two major subdivisions within the classification of MAS, which hitherto 


\section{Key points}

- Dysregulated production of IFN $\gamma$ and other pivotal cytokines including IL-1, IL-6 and IL-18 can arise from either loss or gain of function in the immune response, with different implications for therapy and prognosis.

- Although macrophage activation syndrome (MAS) is often termed autoinflammatory in both loss and gain of function phenotypes, adaptive lineage cells, especially T cells, are clearly important in both settings.

- The distinct MAS phenotype that arises with hypercytokinaemia can be explained by activation of macrophages that are closely juxtaposed to sinusoids that permit direct phagocytic access to haematopoietic lineage cells.

- Recognizing MAS variants and the important anatomical considerations around macrophage perivascular topography in the marrow, liver and spleen allows for a novel evaluation of other states associated with hypercytokinaemia.

- Considering the boundaries of immunodeficiency and immune hypersensitivity in MAS related to systemic juvenile idiopathic arthritis and adult-onset Still disease, and the role of lymphoid cells, severe COVID-19 is generally a distinctive lung-specific immunopathology.

- The proposed model for MAS immune heterogeneity and cellular topography can be applied to understanding other hyper-inflammatory conditions.

has generally been viewed as innate immune-driven or autoinflammatory in nature. We frame this model in the context of cytokine-targeting strategies proposed for the treatment of COVID-19, which we believe could be erroneous because of the conflation of two distinct MAS immunopathologies - one with loss of immune function and the other with gain of immune function. We also consider the cytokine storm phenotype in children with what is termed multisystem inflammatory syndrome in children (MIS-C; also known as paediatric inflammatory multisystem syndrome temporally associated with COVID-19) ${ }^{8}$, which is also distinct from MAS. We also highlight how many viral infections, including SARS-CoV-2, can trigger MAS, but some viral infections trigger a diffuse haemorrhagic disease phenotype that differs from other cytokine storm scenarios and MAS, with a strong bleeding diathesis, but this viral haemorrhagic phenotype is not well understood. Finally, we discuss the emerging data pertaining to sJIA-AOSD spectrum disease in which certain cytokine-directed therapies might not prevent development of MAS.

\section{MAS versus other cytokine storm states}

Initial cytokine storm or hypercytokinaemia states might lead to several discrete clinical scenarios, including MAS, and these scenarios can quickly and concurrently appear or evolve (FIG. 1). Although we highlight differences in these cytokine storm scenarios, the severe cytokine storm associated with superagonistic anti-CD28 antibodies showed rapid and concurrent evolution of different clinical phenotypes ${ }^{9}$ (FIG. 1). Furthermore, the cytokine storm associated with CAR $\mathrm{T}$ cell therapy appears within days; although exhibiting features of MAS, it can be much more extensive with multi-organ involvement ${ }^{10}$ and, as described later, responds differently to anti-IL-6 therapy.

The kinetics of MAS in sJIA-AOSD differs from the aforementioned scenarios, having a generally slower evolution; the earliest phases of active sJIA-AOSD strongly overlap clinically with MAS, reflecting initial hypercytokinaemia that is followed by macrophage haemophagocytosis in the bone marrow, liver, spleen and other sites ${ }^{11,12}$ (FIG. 1a). In some cases of hypercytokinaemia, the predominant picture is of an initial capillary leak syndrome (CLS) with severe oedema, weight gain and hypotension (FIG. 1 b). Unlike MAS, this cytokine storm state is associated with predominant endothelial dysfunction, with a normal (rather than low) haemoglobin concentration, diffuse fluid retention and progressive organ damage $^{13}$ (FIG. 1 b). The CLS state also probably accounts for other manifestations, including cardiac, gastrointestinal and neurological dysfunction, among other scenarios, that are common following CAR $\mathrm{T}$ cell therapy ${ }^{14}$ and that are being reported in MIS- $\mathrm{C}^{15-17}$.

A third hypercytokinaemia scenario that is often, but not exclusively, linked to sepsis without specific evidence of MAS or CLS has been termed systemic inflammatory response syndrome (SIRS). SIRS can progress to multi-organ dysfunction syndrome (MODS), which includes acute respiratory distress syndrome (ARDS $)^{18}$. The SIRS scenario probably reflects the influence of microorganism-mediated activation of organ-resident immune cells via pattern recognition receptors or direct tissue injury via damage-associated molecular pattern-mediated organ damage, distinct from MAS or CLS phenotypes (FIG. 1c). Severe SARS-CoV-2 infection that leads to intra-pulmonary immune activation, including regional but not systemic macrophage activation with associated immunothrombosis, generally fits within this SIRS scenario. However, SARS-CoV-2related hypoxaemia with lung-centric cytokine dysregulation might not trigger considerable elevations in cytokines or systemic inflammatory markers, but can be successfully stratified and treated with corticosteroids on the basis of hypoxia and oxygen requirements ${ }^{19}$. The remarkable degree of pulmonary capillary and adjacent vasculature immunothrombosis probably explains the low frequency of viral nucleic acid detection in the blood $^{20}$, thus minimizing the magnitude of systemic cytokine responses.

Although MAS can be associated with coagulopathy, in another scenario a predominant intravascular MAS-like state exists. This scenario typically occurs in viral haemorrhagic fevers that demonstrate a tropism for monocyte lineage cells, including dengue ${ }^{21}$, influenza and Ebola virus disease ${ }^{22-25}$ (FIG. $1 \mathrm{~d}$ ), in which it has long since been demonstrated that cytokines derived from mononuclear cells, rather than direct endothelial infection, can be the main orchestrator of endothelial damage $^{26}$.

According to the scheme in FIG. 1, cytokine storm features have two major subdivisions: those attributable to cytokines derived from the lymphoid organs (FIGS $1 \mathrm{a}, \mathrm{b}$ ) or cells (FIG. 1d), and from cytokines emerging from infected or damaged non-lymphoid tissues, which could be colloquially described as 'in house' cytokine production (FIG. 1c). In a study of patients with severe inflammation and fatal influenza infection, for example, IL- 6 and IL-1 concentrations were much higher in the diseased lung in comparison with the serum, whereas IFN $\gamma$ levels were modestly elevated and comparable in the serum and lung, probably reflecting the extra-pulmonary lymphoid tissue origins of the latter cytokine ${ }^{27}$. We believe 
a Classical MAS with haemophagocytosis (in liver, spleen, marrow and elsewhere) and coagulopathy

Exogenous cytokines activate haemophagocytosis

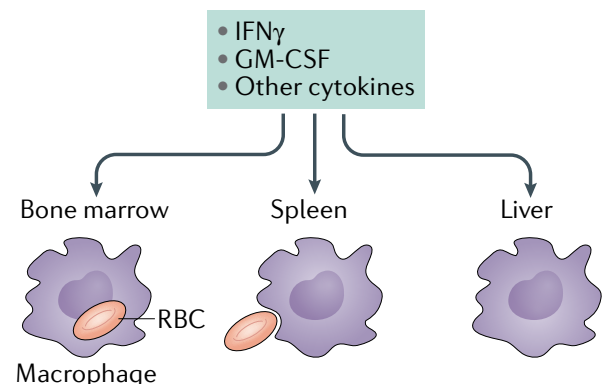

C Multi-organ dysfunction syndrome

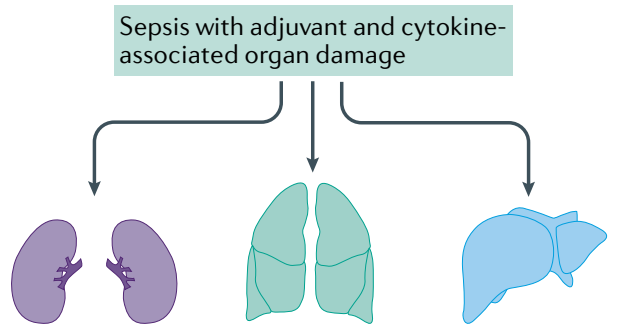

b Cytokine storm leading to CLS

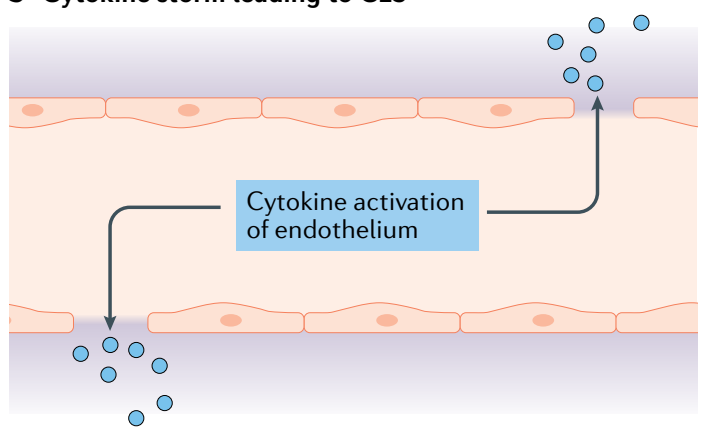

d Viral infection of macrophages with coagulopathy and diffuse bleeding

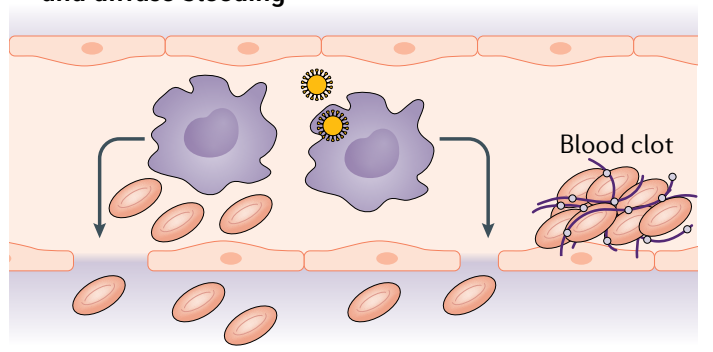

Fig. 1 | The cellular basis for MAS and allied disorders. Cytokine storm can manifest in different ways. It is important to point out that these scenarios can rapidly and simultaneously evolve in some settings in which scenarios division is artificial. a | Where the monocyte system comes into direct contact with the circulation in the liver, spleen and bone marrow, a physiological mechanism exists for the removal of worn red blood cells (RBCs). A predominant dysregulation of this mechanism in the context of a cytokine storm leads to the 'classical' macrophage activation syndrome (MAS) phenotype. This phenotype is associated with severe systemic inflammation and can trigger a disseminated intravascular coagulation (DIC). $\mathbf{b} \mid$ Cytokines predominantly affecting the endothelial system give rise to a different cytokine storm scenario, characterized by a capillary leak syndrome (CLS) that manifests with hypotension, diffuse tissue oedema and hypoalbuminaemia as key features. c | Organ-specific bacterial or viral infection and/or septicaemia can trigger a cytokine storm that can lead to specific dysfunction of that organ (for example, the lung with SARS-Cov-2 infection) and contribute to progressive multi-organ dysfunction without specific features of MAS or CLS. The influence of adjuvant and cytokine release on pattern recognition and cytokine receptors and the site of organ infection can trigger local or systemic inflammation. Unlike the classical MAS pathology, in which lymphoid dysregulation and high levels of IFN $\gamma$ can promote pathology, this scenario might be more strongly linked to tissue-specific dysfunction with comparatively low levels of IFN $\gamma$, as the pathology is more innate immune-driven. The inflammatory state triggered by COVID-19 better fits this scenario of organ-specific dysfunction related to infection. $\mathbf{d}$ |A MAS-like state dominated by coagulopathy due to intravascular viral infection of monocyte lineage cells with cytokine release and prominent clotting cascade activation can exhibit features of DIC. Unlike the DIC of classical MAS, however, this DIC reflects a viral tropism for macrophages. This cytokine storm scenario is typically seen with haemorrhagic viral infection that usually involves circulatory myeloid cells and manifests as diffuse bleeding in addition to MAS-like features. GM-CSF, granulocyte-macrophage colony-stimulating factor.

that terms like MODS and SIRS are applied inappropriately as diagnostic terms in scenarios in which there could be such immune-compartmentalized and immune-associated organ dysfunction. Although these scenarios are more difficult to recognize than MAS or CLS, and using the SIRS and MODS labels could lead to a false sense of security in managing adults and children exclusively with supportive care, whereas immunomodulatory therapies should be considered given the reported inter-relationships between these conditions (that is, SIRS and MODS) and MAS ${ }^{28}$.

\section{Monocyte system topography in MAS}

In physiology, worn-out cells are removed by efferocytosis, in which phagocytic cells clear apoptotic cells. However, effete red blood cells (RBCs), having no nucleus, cannot undergo apoptosis and are physiologically removed by haemophagocytosis, which can occasionally be seen in normal bone marrow examination or during infection. The monocyte system, also known as the reticuloendothelial system, is responsible for removing RBCs and is mainly operational in the bone marrow sinusoids, the liver sinusoids and spleen red pulp sinusoids; anatomically, all of these sites characteristically enable direct contact between macrophages and the circulation (and thus erythrocytes) (FIC. 2a).

The spectrum of inflammation in MAS is typified by severe systemic features that can occur in the absence of discernible organ involvement. Whole-body PET scanning can be normal in the face of severe systemic inflammation ${ }^{29}$, which suggests a high burden of inflammation undetectable by PET, probably reflecting the involvement of dynamically circulating immunocytes especially activated mononuclear phagocytes and 


Bone marrow: loose
arrangement of marrow
sinusoid endothelial cells
enables direct contact
between macrophages
and RBCs
Consequences of MAS:
- Anaemia
- Pancytopenia
Liver: RBC disposal by
Kupffer cells lining the
walls of liver sinusoids
Consequences of MAS:
- Anaemia
- Abnormal liver
function tests
- Elevated triglycerides
- Coagulopathy due to
decreased synthetic
function

Spleen: splenic red pulp with fenestrated venous sinuses and pulp cords (with macrophages) filters RBCs

Consequences of MAS:

- Anaemia

- Pancytopenia

Fig. 2 | The MAS phenotype in relation to physiological erythrocyte disposal. a | Erythrocytes, which lack a nucleus and cannot undergo apoptosis, are physiologically removed by the reticuloendothelial system by macrophages that are strategically juxta-positioned in sinusoids in the bone marrow, liver and splenic red pulp so that they have direct access to circulatory red blood cells (RBCs). The precise basis for why cytokine storm scenarios trigger macrophage activation syndrome (MAS) rather than other scenarios such as capillary leak syndrome or multi-organ dysfunction syndrome remains to be better defined. b | Experimental models b

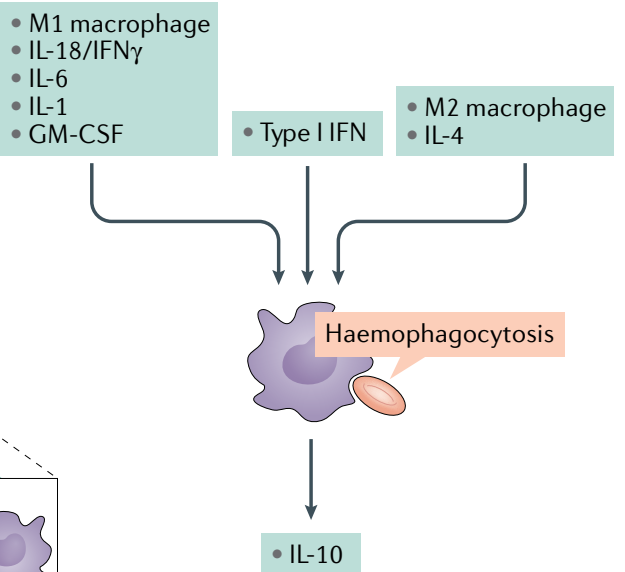

show how the erythrocyte removal system is finely tuned. The normal physiological state of erythrocyte disposal involves M2 macrophage polarization. In experimental settings, perturbations towards T helper 1 $\left(T_{H} 1\right)$ cytokines (including IFN $\gamma$ ) or $T_{H} 2$ cytokines (via excessive IL-4 production) lead to haemophagocytosis, in an exaggeration of the normal physiology. Experimentally, IL-10 production by haemophagocytosing macrophages exerts an anti-inflammatory effect in the local environment, thus representing counterbalancing of haemophagocytosis. GM-CSF, granulocyte-macrophage colony-stimulating factor. neutrophils - and also the fact that such cells are diffusely dispersed in the tissues. Indeed, when ${ }^{18}$ FDG-PET scanning is positive in MAS cases, uptake of FDG is increased in the spleen and marrow, two important sites of effete RBC degradation ${ }^{30}$. Collectively, the MAS pattern of cytokine storm seen in SJIA-AOSD probably reflects progressive anaemia and coagulopathy, generally affording the physician time to recognize it.

\section{Cytokines and haemophagocytosis}

Haemophagocytosis in experimental MAS is orchestrated by cytokines including IFN $\gamma$, GM-CSF, IL-6 and IL-1, with some of these cytokines providing strong autocrine activation of macrophages ${ }^{31-34}$. The first cytokine incriminated in experimental MAS was IFN $\gamma$, in perforin-deficient mice ${ }^{35}$. In experimental murine models other than perforin deficiency, non-immunodeficient mice repeatedly fed a TLR9 agonist or infected with lymphocytic choriomeningitis virus (LCMV) developed haemophagocytosis ${ }^{36}$. Demonstrable haemophagocytosis in the TLR9-induced model was dependent on IL-10 blockade but not on T cells ${ }^{36}$. LCMV-induced haemophagocytosis was dependent on type I interferon, which was readily measurable in the peripheral blood ${ }^{37}$. Such type I interferon-dependent signatures are also an important feature of severe systemic lupus erythematosus (SLE) in humans, which has a well-recognized association with $\mathrm{MAS}^{38}$. However, human SLE-associated MAS has also been linked to
IFN $\gamma^{39}$, the aforementioned key cytokine in the original perforin-deficiency murine models of MAS. With respect to IFN $\gamma$, humans with loss-of-function mutations in the IFN $\gamma$ receptor reportedly develop features of MAS but without haemophagocytosis ${ }^{40}$, thus implicating other cytokines in the phenotype. However, in the aforementioned TLR9-induced MAS model, IFN $\gamma$ knockout mice were protected from anaemia but not from other cytopenias, and still experienced haemophagocytosis following anti-IL-10 therapy ${ }^{41}$.

The normal physiological state of erythrocyte disposal involves M2 macrophage polarization, but further M2 shifting is linked to haemophagocytosis in experimental MAS, in which excessive amounts of IL-4, an archetypal M2 cytokine, exacerbates haemophagocytosis ${ }^{42,43}$, thus illustrating the finely balanced macrophage biology that regulates normal disposal of effete RBCs (FIG. 2b). A particular feature of MAS as compared with sepsis is a CRP-independent elevation of soluble CD163 (haemoglobin scavenger receptor) and CD163 expression in tissues ${ }^{44}$, and it has been suggested that most of the macrophages involved in haemophagocytosis might be predominantly M2 polarized (FIG. 2b). Intriguingly, in the LCMV- and TLR9 ligand-induced models of haemophagocytosis, IL-10 production by haemophagocytosing monocyte-derived dendritic cells was important in mitigating tissue damage and mortality, as either blockade of IL-10 or blockade of haemophagocytosis itself was associated with a worse outcome $\mathrm{e}^{37}$. 


\section{The MAS concept in the context of HLH}

Great insights into MAS have been gleaned from the paediatric haematology community's experience with primary $\mathrm{HLH}$, and this experience has strongly shaped the immunopathogenesis narrative of MAS associated with sJIA-AOSD ${ }^{45}$. The MAS activation in the paediatric arena outside primary HLH is characterized as a form of secondary HLH in paediatric haematology. Primary HLH is a systemic, progressive and potentially fatal autosomal-recessive condition that is genetically and functionally defined by immunodeficiency of the 'killing machinery' of natural killer (NK) cells and cytotoxic $\mathrm{T}$ cells ${ }^{46}$ (FIG. 3). When target cells are not efficiently killed, prolonged immunological synapse formation occurs, resulting in high levels of IFN $\gamma$ production that in turn lead to macrophage activation with haemophagocytosis ${ }^{47}$. A variety of primary $\mathrm{HLH}$-associated genetic mutations that lead to the 'perforinopathies', which are characterized by defective trafficking of cytolytic granules from lymphocytes to target cells, have been described, including PRF1,
LYST, RAB27A, STXBP2, UNC13D and STX11, as well-reviewed elsewhere ${ }^{48-53}$.

The primary HLH narrative, with its intrinsic immunodeficiency rather than immune hypersensitivity, has, in the main, underpinned the entire concept of sJIA and MAS immunopathology. Although primary HLH was originally described as an autosomal-recessive disease of infancy or childhood, it was later shown that $14 \%$ of adults with a primary HLH phenotype had heterozygous hypomorphic mutations in perforin pathway-related genes $^{54}$. In studies testing the theory that cases of sporadic sJIA-related MAS might be in some way partially linked to a similar immunodeficiency state, heterozygous mutations in perforin pathways potentially leading to functional defects have been reported in approximately one-third of patients with MAS in the context of sJIA ${ }^{55-57}$. Also, more than half of uncomplicated cases of sJIA exhibit bone marrow haemophagocytosis, highlighting the close pathophysiological link between sJIA and MAS ${ }^{58}$.

The overarching hypothesis of primary HLH is that failure of $\mathrm{CD}^{+} \mathrm{T}$ cells and NK cells to eliminate virally

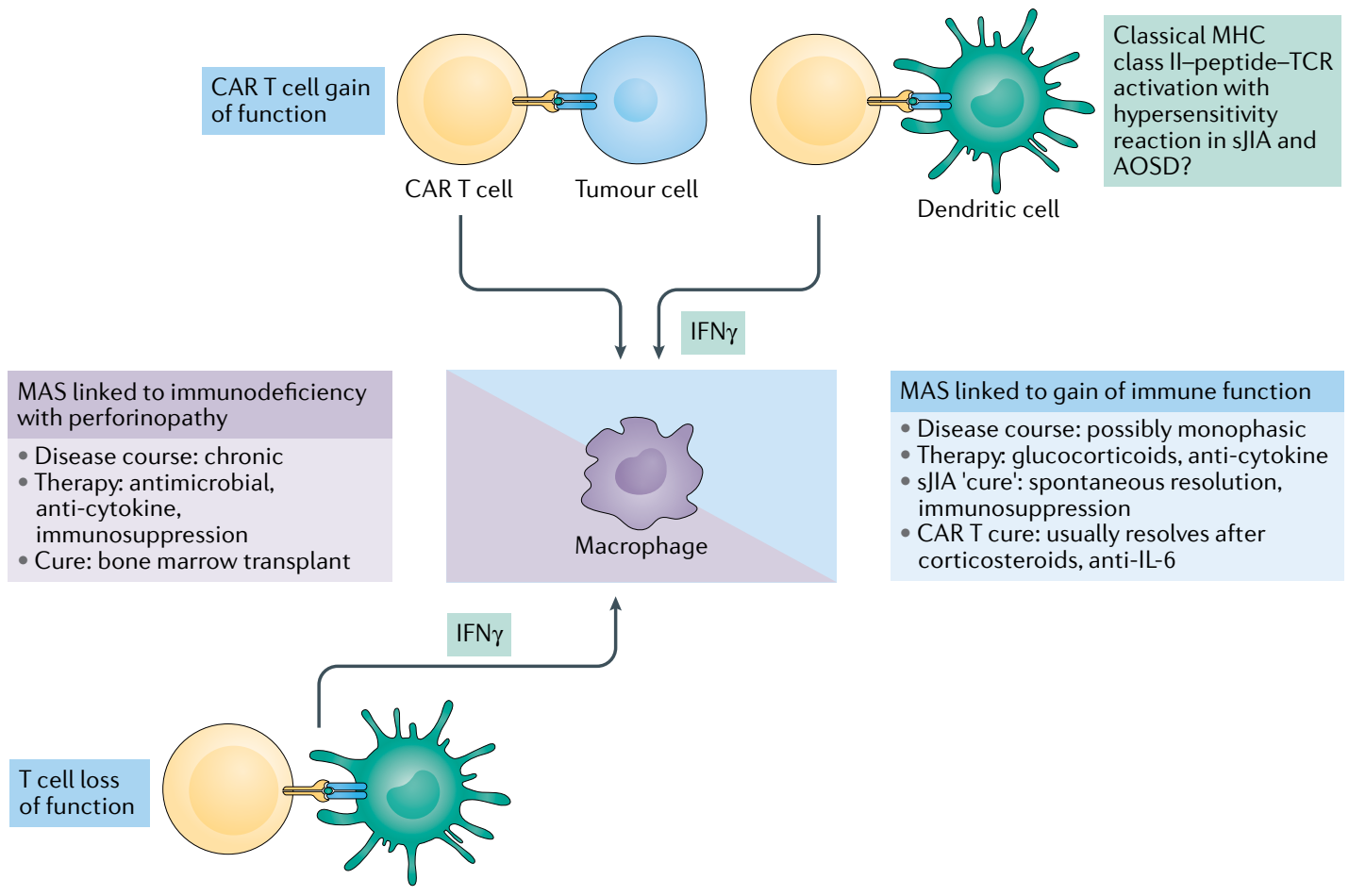

Fig. 3 | MAS spectrum from immunodeficiency to immune hypersensitivity. In classical monogenic primary haemophagocytic lymphohistiocytosis, the failure of the first-line of defence, especially natural killer cells, and also CD8 ${ }^{+}$ T cells, leads to persistence of viral antigens. Inability to remove infected immune cells leads to persistent activation of dendritic cells and other antigen-presenting cells and widespread priming of $C D 8^{+} T$ cells (which again can be ineffective at killing) and also expansion of T helper $1\left(\mathrm{~T}_{H} 1\right) \mathrm{CD} 4^{+} \mathrm{T}$ cell clones. An IFN $\gamma$-driven pathology via hyper-activation of macrophages resulting in collateral damage including haemophagocytosis, liver pathology, coagulopathy and other manifestations; other $\mathrm{T}_{\mathrm{H}} 1$ cytokines, including GM-CSF, could also contribute to macrophage activation. Engineered gain of function as part of chimeric antigen receptor (CAR) T cell therapy leads to a similar but monophasic phenotype that may include macrophage activation syndrome (MAS) phenotype features and that subsides with elimination of the target antigen (cell population). Also, given that immunodeficiency in the perforin pathway is evident in $<40 \%$ of systemic juvenile idiopathic arthritis (sIIA) cases and that sJIA is often associated with MHC-II, it is credible that the sore throat at the onset of sJIA and adult-onset Still disease (AOSD) results from a CD4 ${ }^{+}$helper T-cell hypersensitivity reaction following a quickly eliminated non-specific viral trigger; putative antigens await definition. Overlapping immunopathogenic mechanisms could exist. Despite compelling evidence for adaptive T cell responses in both the gain and loss of function settings, sJIA-related and AOSD-related MAS have been placed into the autoinflammatory or innate immunopathology category of disease, rather than along the immunological disease continuum ${ }^{134}$. 
infected antigen-presenting cells and other immune cells leads to profound secondary activation of many $\mathrm{T}$ cell clones, and there is a shared pathophysiology with secondary HLH and MAS features as seen in sJIA$\mathrm{AOSD}^{17,59}$. In studies of perforin-deficient mice, $\mathrm{CD}^{+}$ T cell production of IFN $\gamma$ is essential for the development of experimental primary $\mathrm{HLH}^{35}$. Likewise, in humans with MAS or secondary HLH, a striking abundance of IFN $\gamma$-expressing $\mathrm{CD}^{+} \mathrm{T}$ cells, and not $\mathrm{CD} 4^{+}$ $\mathrm{T}$ cells, was reported in biopsy-obtained liver tissue ${ }^{60}$. Despite their immunological similarities, the use of a single set of diagnostic criteria for both the primary and secondary forms of HLH is fraught with difficulty ${ }^{61}$. Secondary HLH or MAS in the context of sJIA-AOSD is typically characterized by an initial absence of cytopenias, which could lead to diagnostic delay. With this limitation in mind, revised criteria specifically intended for early and appropriate diagnosis of MAS in sJIA have now been formulated ${ }^{62}$.

\section{Understanding sJIA-associated MAS}

In SJIA-AOSD, the umbrella term of secondary HLH can encompass non-severe disease in which haemophagocytosis is hard to demonstrate, rare or even absent, and the more accurate term MAS is preferred. MAS characteristically occurs in SJIA, which itself has been viewed as an autoinflammatory disease, a thesis that draws strong support from dysregulation of IL- $1 \beta$ and IL-18 and the response to drugs that target these cytokines $^{63,64}$. Findings from genome-wide association studies, initially confirming strong associations between MHC class II and sJIA ${ }^{65}$ and later different MHC class II associations with AOSD in Chinese individuals ${ }^{66}$ and other MHC class II associations in smaller European groups ${ }^{65,67}$ potentially incriminate conventional CD $4^{+}$ helper $\mathrm{T}$ cells and, by implication, $\mathrm{CD} 8^{+} \mathrm{T}$ cells in disease pathogenesis. Notably, a specific link between MAS within the sJIA-AOSD setting and MHC class II associations has not been established to date.

That sJIA is an autoinflammatory disease is widely accepted, a concept that is well justified by the clinical features and responses to IL-1 antagonists ${ }^{68,69}$. However, the aforementioned discovery that MHC class II is associated with sJIA threw "a monkey wrench into the characterization of sJIA as an autoinflammatory disease" and, by implication, $\mathrm{MAS}^{70}$. Unravelling MHC class II associations in humoral autoimmune diseases is facilitated by autoantibody specificity for target antigen pull-down assays and then the ability to identify disease-associated peptides. However, this luxury is not readily available for putative peptides that might be associated with vigorous adaptive immune cell-mediated $\mathrm{CD} 4^{+}$helper $\mathrm{T}$ cell responses that in turn might amplify $\mathrm{CD} 8^{+} \mathrm{T}$ cell responses. Serum biomarkers for sJIA, such as substantial elevation of soluble IL-2 receptor- $\alpha$ subunit (also known as soluble CD25), also strongly indicate T cell hyperactivation $^{71}$.

With CAR T cell therapy, in which T cells are engineered against specific tumour antigens, the ensuing development of cytokine storm with MAS is both proportionate to the tumour volume and related $\mathrm{T}$ cell expansion, powerfully attesting to the theory that gain of function in adaptive immunity, and nothing else, is sufficient and adequate to precipitate a cytokine storm or MAS phenotype ${ }^{72,73}$. This observation lends further credibility to the theory that an immune hypersensitivity reaction originating in an antigen-driven adaptive $\mathrm{CD} 4^{+}$ T cell response could also drive MAS (FIC. 3). Also, MAS in CAR T cell therapy is monophasic and subsides with antigen elimination; sJIA-AOSD may also be monophasic, thus suggesting an antigen-driven response. Given that perforin pathway mutations occur in less than half of MAS cases, that chronic infections are absent in many cases of MAS, and that sJIA and MAS are sometimes monophasic in nature, we propose a simple model to encompass the different dysregulated primary adaptive immune responses that culminate in the predominant autoinflammatory MAS phenotype, with two scenarios in which strategically placed perivascular macrophages (FIG. 2) are central to the dysregulated adaptive immune responses (FIG. 3). A final common pathway of macrophage activation is thus evident, with either loss or gain of $\mathrm{T}$ cell functional activity (FIG. 3).

\section{Classification of the MAS spectrum}

In the previously devised immunological disease continuum of inflammatory disorders, monogenic innate and adaptive immunopathologies represent the extreme boundary conditions ${ }^{74}$. The subsequent introduction of a multidimensional aspect helped to refine this concept, with gain and loss of function in immunity fitting within the extreme boundaries of innate and adaptive immunopathology $y^{75}$. Placing the MAS spectrum along the immunological disease continuum resolves the designation of MAS as autoinflammatory by fully encompassing the role of adaptive immunity and also of loss or gain in immune function ${ }^{74}$.

Accordingly, the full spectrum of MAS phenotypes, beyond the sJIA-AOSD and paediatric HLH disorders, can be accommodated within this scheme (FIG. 4). Just as sJIA and AOSD can have loss or gain of function in innate or adaptive immunity, the same logic can be applied to the classification of MAS in the context of SLE, a disease that has monogenic complement immunodeficiency to immune hyperactivation owing to disordered nucleic acid metabolism ${ }^{76,77}$ (FIG. 4). According to this scheme, it is critical to identify loss of immune function, for which antimicrobial therapy will form an integral part of treatment.

In the oncology arena, MAS and CRS were well recognized before the emergence of CAR $\mathrm{T}$ cell therapy, reported to arise spontaneously in haematological malignancy including myeloid and lymphoid malignancies ${ }^{78}$ and noted to occur in over half of patients with rare intravascular B cell lymphomas ${ }^{79}$. The genesis of MAS in the haematological malignancy setting is complex and is linked to both concomitant infections (including Epstein-Barr virus) and chemotherapy ${ }^{76}$. The assertion that efficient macrophage-mediated removal of effete RBCs is finely tuned is supported by the increasing recognition of myeloid malignancy as a trigger of $\mathrm{MAS}^{78}$ (FIG. 5). MAS associated with various established therapies for malignancies, including chemotherapy and bone marrow transplantation, has 


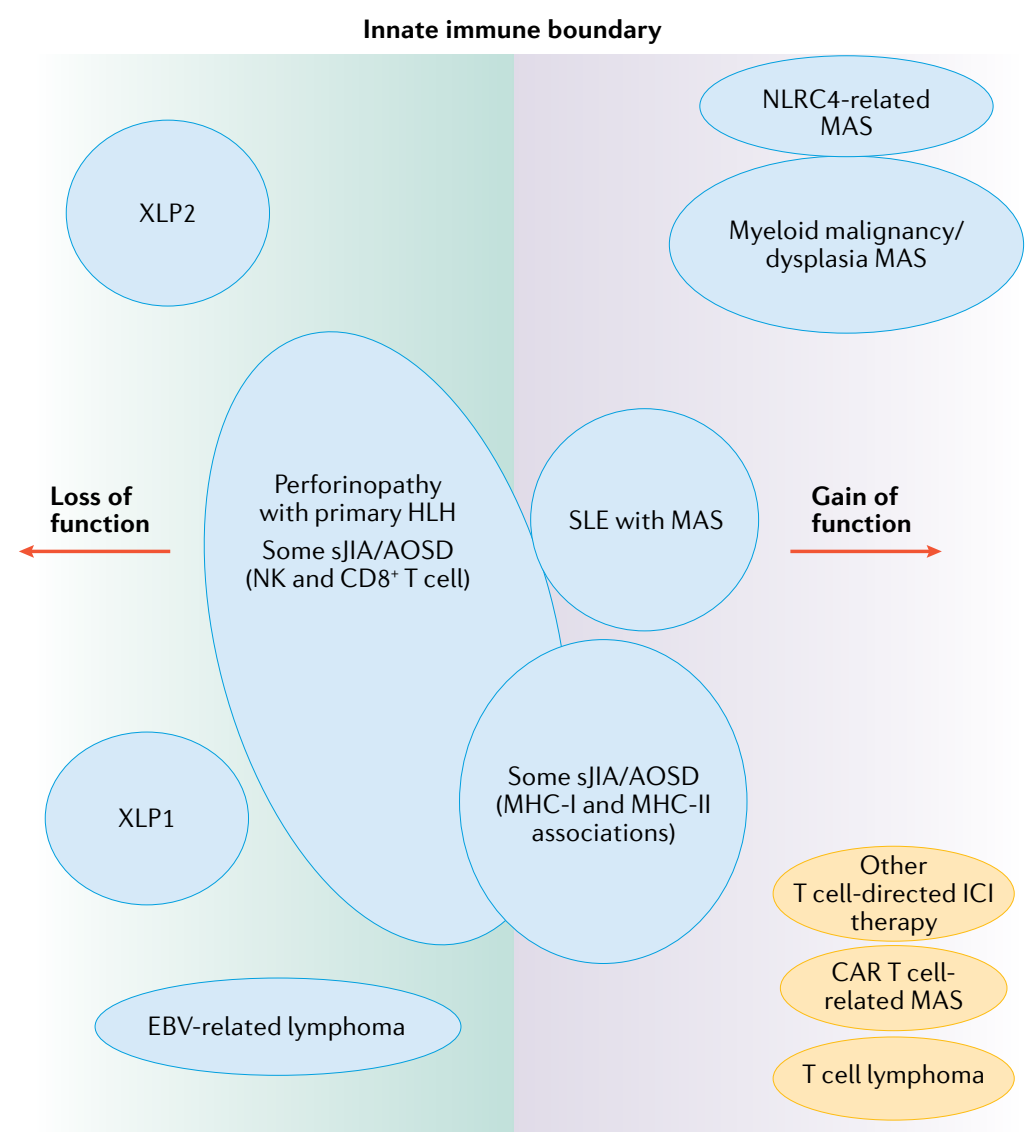

Adaptive immune boundary

Fig. 4 | Classification of the MAS spectrum. The autoinflammatory presentation of macrophage activation syndrome (MAS) can be seen in the context of an integrated immune response. Accordingly, the immunological disease continuum of inflammation against self, modified according to loss and gain of function, can be applied to MAS spectrum disorders. The systemic juvenile idiopathic arthritis (sJIA) and adult-onset Still disease (AOSD) phenotypes span both loss of function and gain of function in innate and adaptive immunity. Likewise, severe inflammation in systemic lupus erythematosus (SLE), another genetically heterogeneous disease, can trigger MAS by mechanisms including both loss and gain of immune function. Rare monogenic disorders leading to MAS can also exhibit loss or gain of immune function. MAS triggered by cancer immunotherapy, including chimeric antigen receptor (CAR) T and bispecific T cell engager therapy, represents a type of gain of function in adaptive immunity. Myeloid malignancy falls in the predominantly innate immune gain of function section. Occasionally, MAS can be triggered in COVID-19, but the dominant mechanism awaits full description. EBV, Epstein-Barr virus; HLH, haemophagocytic lymphohistiocytosis; ICl, immune checkpoint inhibitor; NK, natural killer; XLP, X-linked lymphoproliferative disease.

been ascribed to many factors including dysregulated immune reconstitution and $\mathrm{T}$ cell expansion, as well as infection $^{80-84}$. Experimental MAS in animal models can result from loss of function or gain of function: the relevant animal model with immunodeficiency would be loss of function of $\mathrm{NK}$ and $\mathrm{CD}^{+} \mathrm{T}$ cells $\mathrm{s}^{35}$; with normal immunity, excessive TLR stimulation ${ }^{36}$; and with gain of function, excessive macrophage activation with IL-6 and MAS pathology ${ }^{85}$.

In the haemato-oncology arena, as already mentioned, cytokine storm with MAS is frequent with CAR $\mathrm{T}$ cell therapy and can also occur with bispecific $\mathrm{T}$ cell engagement therapy, with which massive clonal lymphocyte expansion and hypercytokinaemia with MAS features also occur ${ }^{86}$. This MAS scenario is rarely seen with immune checkpoint inhibitor (ICI) therapy, which blocks co-stimulatory signals including CTLA4 and PD1; the ICI strategies affect a comparatively smaller lymphocyte pool that is rarely linked to CRS ${ }^{87}$. These lessons from the clinic illustrate how the CAR $\mathrm{T}$ or bispecific $\mathrm{T}$ cell engagement scenarios with hypercytokinaemia promote a CRS or MAS autoinflammatory phenotype, whereas ICI therapy predominantly unleashes humoral and cellular autoimmune responses that target specific tissues or organs ${ }^{88}$.

\section{MAS insights from translational therapies}

A combination of human genetics and experimental therapeutics in humans is at the forefront of research dissecting the essential mediators of MAS when both the IL-1 pathway and IFN $\gamma$ have already been antagonized ${ }^{2}$. Surprisingly, these studies have already shown different efficacy of key MAS pathway-related cytokines in different clinical settings. Patients with sJIA who have elevated serum concentrations of IFN $\gamma$ or IL-18 (which drive IFN $\gamma$ production by lymphocytes) are reportedly more likely to develop MAS ${ }^{89}$. This finding supports the idea of an MHC class II-peptide-T cell receptor-driven lymphoid tissue response and a critical role for $\mathrm{T}$ cells in MAS development. Likewise, with CAR T cell therapy, the most severe cytokine storm phenotype is linked to extreme hyperferritinaemia and extremely elevated IFN $\gamma$ concentrations (in the $10,000-\mathrm{pg} / \mathrm{ml}$ range), reflecting extensive lymphoid cell activation ${ }^{90}$. IL-12 is an important early driver of IFN $\gamma$ production ${ }^{91}$, but profound elevations and rapid declines in concentrations of this cytokine in severe human CRS probably preclude its antagonism in the clinical setting?.

IFN $\gamma$-activated macrophages produce abundant IL-1, IL-6, GM-CSF and other cytokines. In a 2020 study, emapalumab, an intravenously administered anti-IFN $\gamma$ monoclonal antibody, had some efficacy in the treatment of primary $\mathrm{HLH}^{92}$. There is also some evidence for efficacy of emapalumab for MAS in patients with sJIA, in keeping with our proposed model of loss or gain of function in adaptive immunity and the final common pathway of lymphoid-driven IFN $\gamma$ MAS scenarios ${ }^{93}$ (FIG. 4). However, akin to the human setting, in experimental HLH the MAS phenotype, including haemophagocytosis, can occur in mice deficient in IFN $\gamma$ and perforin; in this model, HLH was associated with neutrophilia but anaemia was absent, GM-CSF was involved and IL-33 blockade was effective ${ }^{94}$.

IL-18 antagonism has also shown preliminary efficacy in proof-of-concept studies in $\mathrm{AOSD}^{95}$, in which subclinical MAS is common ${ }^{58}$. In this setting, macrophages could be important producers of IL-18, which was originally termed IFN $\gamma$-inducing factor; this cytokine could further promote IFN $\gamma$ production by T cells. Given that IL-18 can also be produced by non-immune cells including epithelial cells, keratinocytes and endothelial cells, it could certainly have an important role when the MAS phenotype originates in tissues rather than in immune cells ${ }^{96}$ (FIG. 5). The elevation of IL-18 concentrations in sJIA cases that are destined to progress to MAS needs to be better understood in relationship to lymphoid organ or cell derivation or tissue-specific 


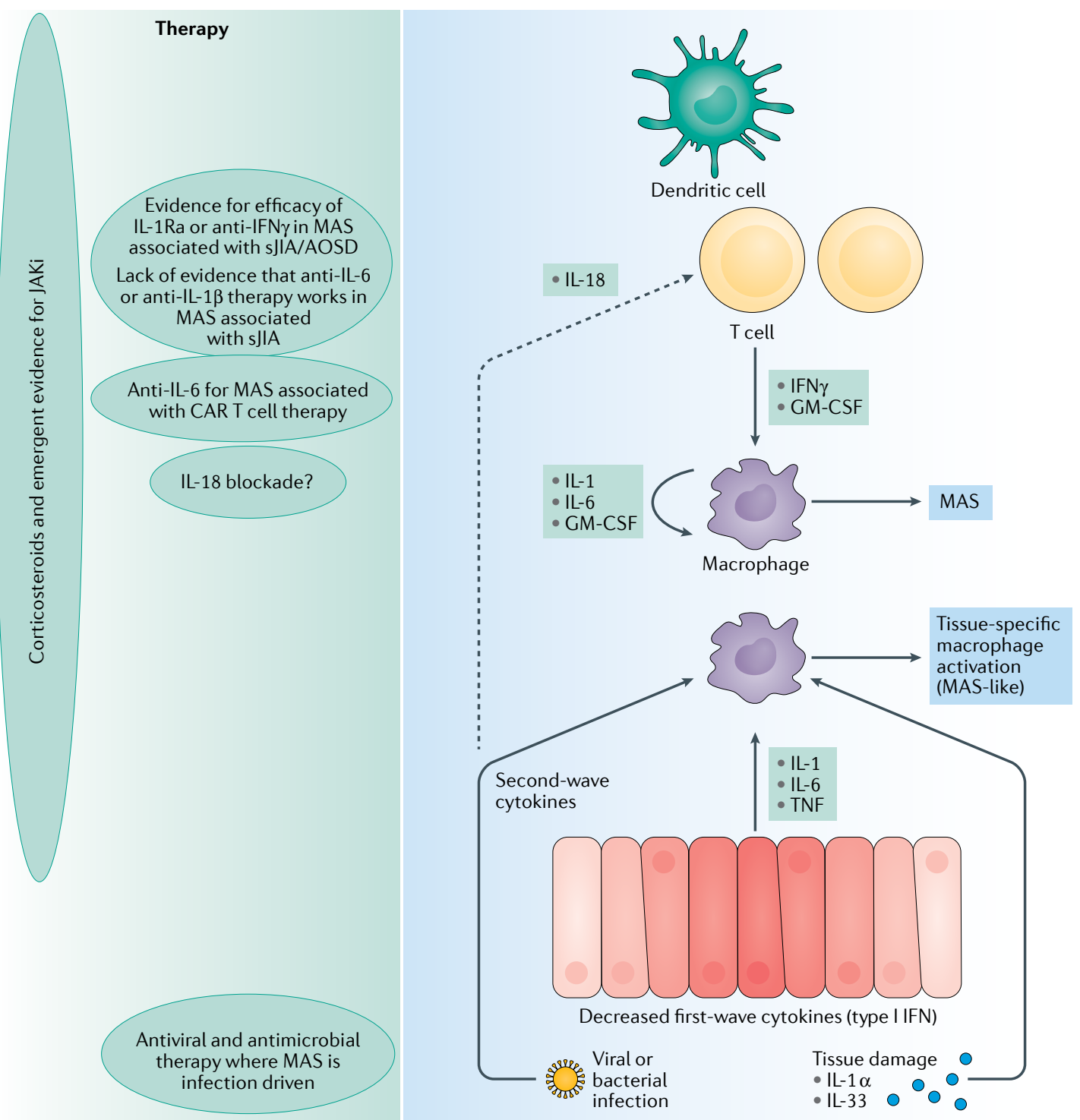

Fig. 5 MAS in the context of experimental therapeutics. In a 'classical' macrophage activation syndrome (MAS) reaction (upper right), antigen-presenting cells and T cells contribute to macrophage activation and hypercytokinaemia. Severe viral infection (by SARS-Cov-2 and other viruses) not controlled by first-wave type I interferon responses (lower right) is associated with strong myeloid infiltration and a second wave of potent pro-inflammatory cytokines, and with strong local immune activation within the lung or other tissues. Many myeloid-related and tissue-derived cytokines, including IL-1, IL-6, TNF and GM-CSF, granulocyte-macrophage colony-stimulating factor (GM-CSF), are elevated in this second wave, but IFN $\gamma$ is not particularly high. Unlike MAS, concentrations of cytokines and ferritin are not particularly elevated, but in some cases of viral infection classical MAS can develop. Treatment of MAS is being empirically defined by experimental medicine. Currently, anti-IL-6 therapy is effective for MAS associated with cancer immunotherapy but not for MAS associated with systemic juvenile idiopathic arthritis (sJIA) or adult-onset Still disease (AOSD). IL-1 receptor antagonist (IL-1Ra) is effective for MAS in sJIA and AOSD and in experimental models of cytokine release syndrome in oncology. IL-1 $\beta$ blockade is effective for sJIA but not for sJIA-associated MAS. IFN $\gamma$ antagonism has shown some efficacy in sJIA-associated MAS and primary haemophagocytic lymphohistiocytosis, but according to this scheme would not be predicted to affect viral pneumonia in most instances. Corticosteroids affect the full spectrum of inflammation associated with sJIA-AOSD to MAS and, given the multi-cytokine and multi-cell inhibition of Janus kinase inhibitors (JAKi) and preliminary emerging data, these agents will also probably have a broad impact. CAR, chimeric antigen receptor.

IL-18 production $^{64,97-99}$. In the autosomal-dominant autoinflammatory disorder caused by NLRC4 mutation, MAS and severe enterocolitis can develop consequent to relevant inflammasome overactivation, with elevated serum concentrations of IL- $1 \beta$ and very high concentrations of IL-18 (REF. ${ }^{100}$ ). The potential origin of IL-18 from outside the immune cell compartment might be a hitherto unappreciated, but important, factor in recognizing MAS where lymphoid dysregulation could be driven from exogenous or tissue-specific release of activating factors such as IL-18 release (FIG. 6a).

The molecular basis of sJIA and therapeutic response to the IL-1 receptor antagonist (IL-1Ra) anakinra indicate that IL-1 has an important role in this setting ${ }^{63}$. 
IL-1 blockade with anakinra, which antagonizes both IL- $1 \alpha$ and IL- $1 \beta$, has shown efficacy in the treatment of MAS associated with sJIA ${ }^{101-105}$. Some preliminary evidence of the benefit of IL-1Ra for infections, including dengue and other viral infections, has also been reported $^{106}$. Conversely, canakinumab, which solely blocks IL-1 $\beta$, failed to prevent MAS, implicating that IL- $1 \alpha$ is important in the disease ${ }^{107}$ (FIC. 5). The basis for the pivotal role of the IL-1 pathway in MAS is incompletely understood, but many factors, including the role of IL- $1 \beta$ in augmenting IL-12-induced production of IFN $\gamma$ in NK cells in experimental models, could be relevant $^{108,109}$.

Anti-IL-6 therapy has also shown efficacy in sJIA$\mathrm{AOSD}$, conditions that are forerunners of $\mathrm{MAS}^{45}$. In the immune-oncology literature, the impressive resolution of CAR T cell therapy-related cytokine storm and MAS achieved with corticosteroids or anti-IL-6 therapy has also served as an indication that such strategies could be considered for severe COVID-19 pneumonia $^{110}$. The use of the IL- 6 receptor antagonist tocilizumab in MAS associated with CAR T cell therapy is virtually curative; a

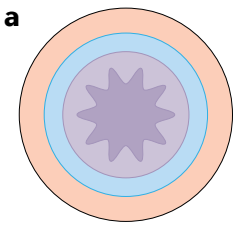

Intestinal epithelium

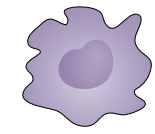

Macrophage

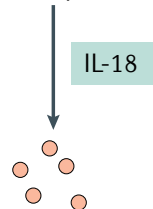

d

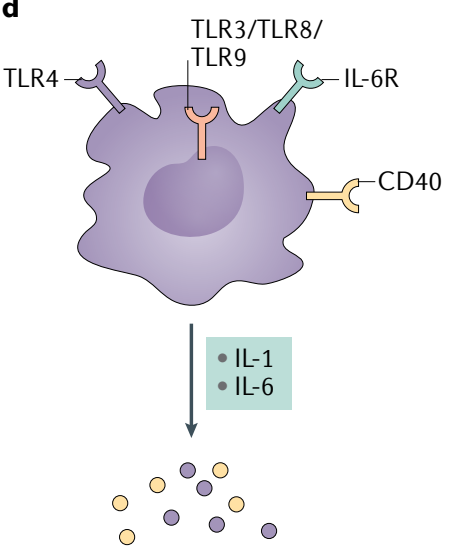

First wave

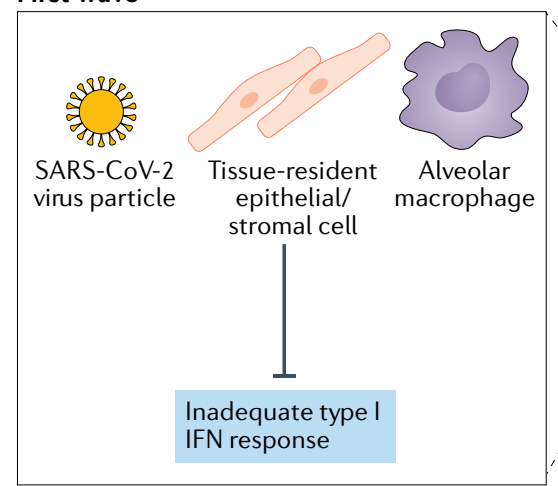

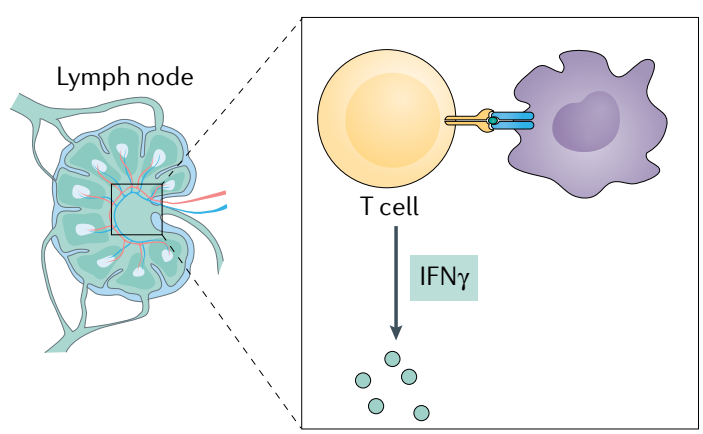

Fig. 6 | Tissue basis for cytokine storm phenotypes encompassing COVID-19. a | Dysregulation of inflammasomes and particularly NLRC4 leads to excessive production of IL-1 $\beta$ and IL-18 by intestinal epithelial cells and myeloid cells, causing generalized antigen-independent $T$ cell expansion with production of IFN $\gamma$ and other cytokines and a macrophage activation syndrome (MAS)-like pattern as well as intestinal pathology reflecting dysregulated intestinal mucosal cytokine production. b | Myeloid or lymphoid cell transformation can lead to MAS. Myeloid malignancy with associated gain of function can contribute to a MAS-like picture. Likewise, gain of function in the lymphoid system, as in lymphoid malignancy or lymphoid viral infection associated with immunodeficiency and transformation, can also activate macrophages. $\mathbf{c}$ A cytokine release syndrome leading to MAS can occur in the setting of perforinopathyassociated immunodeficiency. Likewise, the immune activation that occurs with a hypersensitivity reaction to an infectious trigger has the same effect, with excessive production of IFN $\gamma$ and other cytokines with activation of macrophages. Based on lessons from the monophasic disease linked with chimeric antigen receptor T cell therapy and from the associations of MHC II with systemic juvenile idiopathic arthritis (s)IA) and adult-onset Still disease (AOSD), some of the MAS phenotypes encountered in rheumatology, including cases of MAS associated with sJIA, AOSD and systemic lupus erythematosus, fit with this lymphoid tissue-originating disease. $\mathbf{d} \mid$ A cytokine release syndrome with MAS can occur in the setting of excessive stimulation of Toll-like receptors (TLRs), including TLR9 and TLR4 stimulation in IL-6 overexpression models. The experimental phenotype may correlate with severe sepsis in humans, including viral and bacterial sepsis, which strongly and directly stimulates myeloid cells. Gain-of-function mutations related to innate immunity and factors such as inflammaging could drive this pattern of cytokine storm, which is more organ-centric. Viral infections with a tropism for mononuclear cells or macrophages, such as haemorrhagic virus, could lead to this scenario. e I Immune cell activation, as shown in part $\mathbf{d}$, in a setting such as severe COVID-19, could drive organ- or tissue-specific damage rather than lymphoid or myeloid pathology changes seen in MAS. Severe localized organ-specific inflammation could be responsive to anti-inflammatory strategies, even though the compartmentalized inflammatory response might not trigger marked hypercytokinaemia. Parts $\mathbf{b}, \mathbf{d}$ and $\mathbf{e}$ also illustrate how viral, pathogenic cell or organ tropism profoundly influences the disease phenotype. The tropism of the SARS-CoV-2 virus for the alveolus determines what is generally a very distinct disease phenotype that may occasionally be associated with a more typical MAS phenotype evolution. 
however, this effect might coincide with elimination of the target antigen (FIG. 5). Conversely, tocilizumab therapy in sJIA has been linked to MAS evolution, yet again illustrating how the clinical use of cytokine antagonism is dissecting human immunopathology and providing immune cartography of human disease ${ }^{111-113}$. These findings from tocilizumab trials indicate there are clear differences between MAS in the context of sJIA and CAR $\mathrm{T}$ cell therapy. In murine models, IL- 1 has been shown to have a key role in CAR T cell therapy, and in the experimental setting IL-1Ra has been associated with excellent responses $^{72,114}$

Like corticosteroids, Janus kinase (JAK) inhibitors have direct and broad immunosuppressive effects on cytokines and immune cells, and are promising emergent MAS therapies (FIG. 5). In a small study, the JAK1-JAK2 inhibitor ruxolitinib showed promising efficacy in adults with secondary $\mathrm{HLH}^{115}$. Other JAK inhibitors, including tofacitinib and baricitinib, have also demonstrated some efficacy in treatment-refractory sJIA or AOSD ${ }^{116,117}$.

\section{COVID-19 in context}

Having provided a framework for the spectrum of innate and adaptive immunity in the context of gain or loss of immune function in MAS, here, we briefly look at COVID-19 infection through this lens. At the start of the COVID-19 pandemic, it quickly emerged that many cases of severe or critical COVID-19 had features of cytokine storm, including fever, marked elevations in acute phase responses and coagulopathy ${ }^{118}$. As the spectrum of clinical manifestations with COVID-19 became apparent, it was clear that a subset of patients had substantial morbidity and mortality owing to a 'hyperinflammatory syndrome', even though differences from typical MAS existed ${ }^{119}$. Unfortunately, a homogeneous view of 'cytokine storm syndrome' and MAS with extrapolations from CAR T cell therapy might have erroneously contributed to overoptimism regarding the use of immunosuppression and cytokine-targeting strategies, something that we previously highlighted ${ }^{120}$ (FIG. 6).

Given the aforementioned limitations of measuring cytokines for the diagnosis of a cytokine storm, use of the HScore ${ }^{121}$, which was developed for the recognition of secondary HLH or MAS in cohorts that included patients with rheumatological diseases (such as sJIA and SLE), has been suggested as a way of recognizing cytokine storm in COVID-19 pneumonia ${ }^{122}$. The magnitudes of hyperferritinaemia and elevations in CRP concentrations evident in critical COVID-19 infection are modest compared with those typically seen in MAS ${ }^{123}$. The cytokine storm in COVID-19 pneumonia also has rather underwhelming elevations in concentrations of pivotal cytokines, including IL- 6 , thus indicating a lung-specific injury rather than a typical cytokine storm scenario $^{122,124}$ (FIG. 6e). Indeed, the overall clinicopathological characterization of severe COVID-19 infection has many substantial differences from $\mathrm{MAS}^{120}$. A notable difference from cytokine storm in MAS and its associated DIC is that the COVID-19 lung-centric macrophage activation often occurs without other features of MAS and is associated with a pulmonary immunothrombosis that has been termed pulmonary intravascular coagulopathy (PIC), in contradistinction to $\mathrm{DIC}^{125}$. By 'setting up shop' in the alveolar network, with subsequent lung-centric immunothrombosis, SARS-CoV-2 is distinct from other events that incite cytokine storm and provides a robust framework for understanding the increased mortality of COVID-19 pneumonia in certain ethnic groups ${ }^{20,126}$.

Previous studies of influenza demonstrated that fatal disease was associated with IL- $1 \beta$ and IL- 6 concentrations that were 1,000-fold higher in bronchoalveolar lavage fluid than in blood, whereas IFN $\gamma$ concentrations were only slightly elevated and were of the same magnitude in the lung and serum, pointing to a probable lung-specific endogenous cytokine release as the main promoter of disease, distinct from the commonest MAS groups in sJIA-AOSD, which is a lymphoid cell-driven MAS $^{27}$ (FIG. 6). According to this model, severe pulmonary-centric COVID-19-related inflammation that is likewise associated with low IFN $\gamma$ concentrations would not be amenable to IFN $\gamma$ blockade unless florid MAS features, including profound hyperferritinaemia, were superimposed.

Targeting other cytokines relevant in MAS might similarly lack efficacy given that the main issue in COVID-19 pneumonia is severe tissue infection and multifaceted tissue-specific cytokine responses, rather than systemic macrophage activation (FIG. 5). As already described, the features of COVID-19 pneumonia include much lower concentrations of cytokines and ferritins in general compared with MAS, which suggests organ-specific lung disease with a mechanism that is probably shared with infection agents across the SIRSMODS spectrum (FIG. 1). Data supporting the efficacy of corticosteroids for COVID-19 (REF. ${ }^{127}$ ) attest to a severe lung-specific and mostly lung-originating cytokine dysregulation with 'local cytokine flooding' rather than a global cytokine storm (FIG. 6e).

Many viral and other pathogens can trigger MAS, although herpes family viruses are most strongly linked to an MAS phenotype, especially in the perforinopathies. With respect to severe viral pneumonia, whole-exome sequencing in fatal cases of $\mathrm{H} 1 \mathrm{~N} 1$ influenza pneumonia that had features of HLH or MAS antemortem revealed heterozygous mutations in perforin pathway genes, which link the aforementioned $\mathrm{NK}$ and $\mathrm{CD}^{+} \mathrm{T}$ cell toxicity to a hyperinflammatory state ${ }^{128}$. There is compelling evidence that the type I interferon response is disabled, both in the lung and systemically by severe COVID-19 infection ${ }^{129}$. Indeed, critical COVID-19 pneumonia in young sibling pairs has been linked to TLR7 loss of function, which incriminates loss of type I interferon rather than the perforinopathy scenario reported in $\mathrm{HLH}^{130}$. This break in the 'first wave' of anti-viral immunity is linked to the emergence of a severe 'second wave' of innate immune cytokines that leads to severe pneumonia and immunothrombosis (FIG. 6). The kinetics of viral infection in humans, and the precise timing of the second wave of cytokines overcoming it or whether it abates naturally when permissive cells have succumbed or recovered, is unclear, but in simian models the SARS-CoV-2 spike protein could be detected in monkeys succumbing to viral pneumonia with ARDS $^{131}$ (FIG. 6). 
Where does MIS-C, which can share some MAS features, fit in? The pathophysiology of MIS-C generally lacks the severe pulmonary ARDS phenotype typically seen in adults with COVID-19 (REF. ${ }^{132}$ ). Children with MIS-C predominantly manifest a phenotype that is characterized by high concentrations of inflammatory markers and shock with reduced ejection fraction, but lack the hyperferritinaemia, anaemia and coagulopathy that is seen in $\mathrm{MAS}^{133}$. The cytokine storm in this group manifests in a different manner; theories including superantigenic activation of $\mathrm{T}$ cells with a cytokine storm distinct from that seen in MAS have been proposed ${ }^{134}$. Some children with MIS-C also develop coronary artery aneurysms, similar to Kawasaki disease, which has been linked to previous viral infection ${ }^{135}$. Interestingly, it is increasingly recognized that a proportion of children with Kawasaki disease also develop MAS-like features ${ }^{136}$. The basis for these distinct and overlapping cytokine storm features remains enigmatic.

\section{Conclusions}

MAS is one discrete outcome of the cytokine storm scenario, and in this Review we have contextualized its unique features and those that overlap with other cytokine storm scenarios. We have summarized how MAS arises as a consequence of perturbation to the dynamics of cytokines that affect RBC clearance. We have also pointed out that a dichotomy exists between the 'perforinopathy theory' of MAS and the large body of evidence incriminating adaptive immune activation in many situations in which MAS arises, such as in sJIA and cancer immunotherapy. Extrapolating this scheme to COVID-19 infection explains the emerging data, demonstrating that immunosuppression does not have a tremendous effect in severe COVID-19 as compared with severe MAS associated with CAR T cell therapy or sJIA-related MAS. We have also highlighted how cytokine storm scenarios can arise from a target organ, such as the lung, or from the primary and secondary lymphoid organs that house lymphocytes. We propose a mechanistic classification of MAS whereby disease can be contextualized along the immunological disease continuum adjusted for loss or gain of function. Dysregulated innate or adaptive immunity in SARS-CoV-2 infection might occasionally trigger an MAS picture, which awaits full molecular elucidation.

Published online 5 February 2021
1. Tisoncik, J. R. et al. Into the eye of the cytokine storm. Microbiol. Mol. Biol. Rev. 76, 16-32 (2012).

2. Crayne, C. B., Albeituni, S., Nichols, K. E. \& Cron, R. O. The immunology of macrophage activation syndrome. Front. Immunol. 10, 119 (2019).

3. Schulert, G. S. \& Grom, A. A. Macrophage activation syndrome and cytokine-directed therapies. Best Pract. Res. Clin. Rheumatol. 28, 277-292 (2014).

4. Schulert, G. S. \& Grom, A. A. Pathogenesis of macrophage activation syndrome and potential for cytokine-directed therapies. Annu. Rev. Med. 66, 145-159 (2015)

5. Behrens, E. M. Macrophage activation syndrome in rheumatic disease: what is the role of the antigen presenting cell? Autoimmun. Rev. 7, 305-308 (2008).

6. Freeman, H. \& Ramanan, A. Review of haemophagocytic lymphohistiocytosis. Arch. Dis. Child. 96, 688-693 (2011).

7. Dimopoulos, G. et al. Favorable anakinra responses in severe COVID-19 patients with secondary hemophagocytic lymphohistiocytosis. Cell Host Microbe 28, 117-123.e1 (2020).

8. Ramcharan, T. et al. Paediatric inflammatory multisystem syndrome: temporally associated with SARS-CoV-2 (PIMS-TS): cardiac features, management and short-term outcomes at a UK tertiary paediatric hospital. Pediatric Cardiol. 41, 1391-1401 (2020).

9. Suntharalingam, G. et al. Cytokine storm in a phase 1 trial of the anti-CD28 monoclonal antibody TGN 1412. N. Engl. J. Med. 355, 1018-1028 (2006).

10. Neelapu, S. S. et al. Axicabtagene Ciloleucel CAR T-cell therapy in refractory large B-cell lymphoma. N. Engl. J. Med. 377, 2531-2544 (2017).

11. Canna, S. W. \& Behrens, E. M. Making sense of the cytokine storm: a conceptual framework for understanding, diagnosing, and treating hemophagocytic syndromes. Pediatr. Clin. North Am. 59, 329-344 (2012).

12. Kelly, A. \& Ramanan, A. V. Recognition and management of macrophage activation syndrome in juvenile arthritis. Curr. Opin. Rheumatol. 19, 477-481 (2007)

13. Siddall, E., Khatri, M. \& Radhakrishnan, J. Capillary leak syndrome: etiologies, pathophysiology, and management. Kidney Int. 92, 37-46 (2017).

14. Shimabukuro-Vornhagen, A. et al. Cytokine release syndrome. J. Immunother. Cancer 6, 56 (2018).

15. Feldstein, L. R. et al. Multisystem inflammatory syndrome in US children and adolescents. N. Engl. J. Med. 383, 334-346 (2020)

16. Whittaker, E. et al. Clinical characteristics of 58 children with a pediatric inflammatory multisystem syndrome temporally associated with SARS-CoV-2. JAMA 324, 259-269 (2020).

17. Dufort, E. M. et al. Multisystem inflammatory syndrome in children in New York State. N. Engl. J. Med. 383 347-358 (2020)

18. Rangel-Frausto, M. S. et al. The natural history of the systemic inflammatory response syndrome (SIRS): a prospective study. JAMA 273, 117-123 (1995).

19. Quartuccio, L. et al. Profiling COVID-19 pneumonia progressing into the cytokine storm syndrome: results from a single Italian centre study on tocilizumab versus standard of care. J. Clin. Virol. 129, 104444 (2020).

20. Fox, S. E. et al. Pulmonary and cardiac pathology in African American patients with COVID-19: an autopsy series from New Orleans. Lancet Respir. Med. 8, 681-686 (2020)

21. Henter, J.-I., Palmkvist-Kaijser, K., Holzgraefe, B. Bryceson, Y. T. \& Palmér, K. Cytotoxic therapy for severe swine flu A/H1N 1 . Lancet 376, 2116 (2010).

22. Henter, J. Can cytostatics be effective in severe avian influenza? The disease picture has similarities with hemophagocytic lymphohistiocytosis. Lakartidningen 103, 1180 (2006)

23. Henter, J.-I., Chow, C.-B., Leung, C.-W. \& Lau, Y.-L. Cytotoxic therapy for severe avian influenza A (H5N1) infection. Lancet 367, 870-873 (2006).

24. Feldmann, H. \& Geisbert, T. W. Ebola haemorrhagic fever. Lancet 377, 849-862 (2011).

25. Cron, R. Q., Behrens, E. M., Shakoory, B., Ramanan, A. V. \& Chatham, W. W. Does viral hemorrhagic fever represent reactive hemophagocytic syndrome? J. Rheumatol. 42, 1078-1080 (2015).

26. Feldmann, H. et al. Filovirus-induced endothelial leakage triggered by infected monocytes/ macrophages. J. Virol. 70, 2208-2214 (1996)

27. Wang, Z. et al. Early hypercytokinemia is associated with interferon-induced transmembrane protein-3 dysfunction and predictive of fatal H7N9 infection. Proc. Natl Acad. Sci. USA 111, 769-774 (2014).

28. Castillo, L. \& Carcillo, J. Secondary hemophagocytic lymphohistiocytosis and severe sepsis/systemic inflammatory response syndrome/multiorgan dysfunction syndrome/macrophage activation syndrome share common intermediate phenotypes on a spectrum of inflammation. Pediatr. Crit. Care Med. 10, 387-392 (2009).

29. Schönau, V. et al. The value of 18F-FDG-PET/CT in identifying the cause of fever of unknown origin (FUO) and inflammation of unknown origin (IUO): data from a prospective study. Ann. Rheum. Dis. 77, 70-77 (2018).
30. Choe, J.-Y., Chung, D. S., Park, S.-H., Kwon, H.-H. \& Kim, S.-K. Clinical significance of 18 F-fluorodexoxyglucose positron emission tomography in patients with adult-onset Still's disease: report of two cases and review of literatures. Rheumatol. Int. 30 1673-1676 (2010).

31. Chomarat, P., Banchereau, J., Davoust, J. \& Palucka, A. K. IL-6 switches the differentiation of monocytes from dendritic cells to macrophages. Nat. Immunol. 1, 510-514 (2000)

32. Hamilton, J. A. GM-CSF in inflammation and autoimmunity. Trends Immunol. 23, 403-408 (2002)

33. Martinez, F. O., Sica, A., Mantovani, A. \& Locati, M. Macrophage activation and polarization. Front. Biosci. 13, 453 (2008)

34. Fernando, M. R., Reyes, J. L., lannuzzi, J., Leung, G \& McKay, D. M. The pro-inflammatory cytokine, interleukin-6, enhances the polarization of alternatively activated macrophages. PLoS ONE 9, e94188 (2014).

35. Jordan, M. B., Hildeman, D., Kappler, J. \& Marrack, P. An animal model of hemophagocytic lymphohistiocytosis (HLH): $\mathrm{CD} 8^{+} \mathrm{T}$ cells and interferon gamma are essential for the disorder. Blood 104, 735-743 (2004).

36. Behrens, E. M. et al. Repeated TLR9 stimulation results in macrophage activation syndrome-like disease in mice. J. Clin. Invest. 121, 2264-2277 (2011).

37. Ohyagi, $\mathrm{H}$. et al. Monocyte-derived dendritic cells perform hemophagocytosis to fine-tune excessive immune responses. Immunity 39, 584-598 (2013)

38. Parodi, A. et al. Macrophage activation syndrome in juvenile systemic lupus erythematosus: a multinational multicenter study of thirty-eight patients. Arthritis Rheum. 60, 3388-3399 (2009)

39. Usami, M. et al. Extensive serum biomarker analysis in patients with macrophage activation syndrome associated with systemic lupus erythematosus. Clin. Immunol. 208, 108255 (2019).

40. Tesi, B. et al. Hemophagocytic lymphohistiocytosis in 2 patients with underlying IFN- $\gamma$ receptor deficiency. J. Allergy Clin. Immunol. 135, 1638-1641 (2015).

41. Canna, S. W. et al. Interferon- $\gamma$ mediates anemia but is dispensable for fulminant Toll-like receptor 9 induced macrophage activation syndrome and hemophagocytosis in mice. Arthritis Rheum. 65, 1764-1775 (2013).

42. Milner, J. D. et al. Sustained IL-4 exposure leads to a novel pathway for hemophagocytosis, inflammation, and tissue macrophage accumulation. Blood 116 2476-2483 (2010).

43. Luzina, I. G. et al. Regulation of inflammation by interleukin-4: a review of "alternatives". J. Leukoc. Biol. 92, 753-764 (2012). 
44. Schaer, D. J. et al. Soluble hemoglobin-haptoglobin scavenger receptor CD163 as a lineage-specific marker in the reactive hemophagocytic syndrome. Eur. J. Haematol. 74, 6-10 (2005).

45. Grom, A. A., Horne, A. \& De Benedetti, F. Macrophage activation syndrome in the era of biologic therapy. Nat. Rev. Rheumatol. 12, 259-268 (2016).

46. Schmid, J. P. et al. Inherited defects in lymphocyte cytotoxic activity. Immunol. Rev. 235, 10-23 (2010)

47. Jenkins, M. R. et al. Failed CTL/NK cell killing and cytokine hypersecretion are directly linked through prolonged synapse time. J. Exp. Med. 212, 307-317 (2015)

48. Chinn, I. K. et al. Genetic and mechanistic diversity in pediatric hemophagocytic lymphohistiocytosis. Blood 132, 89-100 (2018).

49. Stadt, U. Z. et al. Mutation spectrum in children with primary hemophagocytic lymphohistiocytosis: molecular and functional analyses of PRF1, UNC13D, STX11, and RAB27A. Hum. Mutat. 27, 62-68 (2006).

50. Ishii, E. Hemophagocytic lymphohistiocytosis in children: pathogenesis and treatment. Front. Pediatr. 4, 47 (2016)

51. Allen, C. E. \& McClain, K. L. fHLH: becoming a blended family. Blood 124, 1210-1211 (2014).

52. Allen, C. E. \& McClain, K. L. Pathophysiology and epidemiology of hemophagocytic lymphohistiocytosis. Hematology 2015, 177-182 (2015).

53. Schulert, G. S. \& Cron, R. Q. The genetics of macrophage activation syndrome. Genes Immun. 21 , 169-181 (2020)

54. Zhang, K. et al. Hypomorphic mutations in PRF1, MUNC13-4, and STXBP2 are associated with adultonset familial HLH. Blood 118, 5794-5798 (2011)

55. Vastert, S. J. et al. Mutations in the perforin gene can be linked to macrophage activation syndrome in patients with systemic onset juvenile idiopathic arthritis. Rheumatology 49, 441-449 (2010)

56. Bracaglia, C. et al. Mutations of familial hemophagocytic lymphohistiocytosis (FHL) related genes and abnormalities of cytotoxicity function tests in patients with macrophage activation syndrome (MAS) occurring in systemic juvenile idiopathic arthritis (sJIA). Pediatric Rheumatol. 12, 1-2 (2014).

57. Kaufman, K. M. et al. Whole-exome sequencing reveals overlap between macrophage activation syndrome in systemic juvenile idiopathic arthritis and familial hemophagocytic lymphohistiocytosis. Arthritis Rheumatol. 66, 3486-3495 (2014).

58. Behrens, E. M., Beukelman, T., Paessler, M. $\bar{\alpha}$ Cron, R. Q. Occult macrophage activation syndrome in patients with systemic juvenile idiopathic arthritis. J. Rheumatol. 34, 1133-1138 (2007)

59. Rosado, F. G. \& Kim, A. S. Hemophagocytic lymphohistiocytosis: an update on diagnosis and pathogenesis. Am. J. Clin. Pathol. 139, 713-727 (2013).

60. Billiau, A. D., Roskams, T., Van Damme-Lombaerts, R., Matthys, P. \& Wouters, C. Macrophage activation syndrome: characteristic findings on liver biopsy illustrating the key role of activated, IFN- $\gamma$-producing lymphocytes and IL-6-and TNF- $\alpha$-producing macrophages. Blood 105, 1648-1651 (2005)

61. Sen, E. S., Steward, C. G. \& Ramanan, A. V. Diagnosing haemophagocytic syndrome. Arch. Dis. Child. 102, 279-284 (2017).

62. Ravelli, A. et al. 2016 Classification criteria for macrophage activation syndrome complicating systemic juvenile idiopathic arthritis: a European League Against Rheumatism/American College of Rheumatology/Paediatric Rheumatology International Trials Organisation Collaborative Initiative. Ann Rheum. Dis. 75, 481-489 (2016).

63. Pascual, V., Allantaz, F., Arce, E., Punaro, M. \& Banchereau, J. Role of interleukin-1 (IL-1) in the pathogenesis of systemic onset juvenile idiopathic arthritis and clinical response to IL- 1 blockade. J. Exp. Med. 201, 1479-1486 (2005).

64. Yasin, S. et al. IL-18 as a biomarker linking systemic juvenile idiopathic arthritis and macrophage activation syndrome. Rheumatology 59, 361-366 (2020).

65. Ombrello, M. J. et al. HLA-DRB $1 * 11$ and variants of the MHC class II locus are strong risk factors for systemic juvenile idiopathic arthritis. Proc. Natl Acad. Sci. USA 112, 15970-15975 (2015)

66. Li, Z. et al. Both HLA class I and II regions identified as genome-wide significant susceptibility loci for adult-onset Still's disease in Chinese individuals. Ann. Rheum. Dis. 79, 161-163 (2020).

67. Ombrello, M. J. et al. Genetic architecture distinguishes systemic juvenile idiopathic arthritis from other forms of juvenile idiopathic arthritis: clinical and therapeutic implications. Ann. Rheum. Dis. 76, 906-913 (2017).

68. Savic, S., Caseley, E. A. \& McDermott, M. F. Moving towards a systems-based classification of innate immune-mediated diseases. Nat. Rev. Rheumatol. 16 222-237 (2020)

69. Martini, A. It is time to rethink juvenile idiopathic arthritis classification and nomenclature. Ann. Rheum. Dis. 71, 1437-1439 (2012).

70. Nigrovic, P. A. Autoinflammation and autoimmunity in systemic juvenile idiopathic arthritis. Proc. Natl Acad. Sci. USA 112, 15785-15786 (2015)

71. Lin, M. et al. Clinical utility of soluble interleukin-2 receptor in hemophagocytic syndromes: a systematic scoping review. Ann. Hematol. 96, 1241-1251 (2017).

72. Giavridis, T. et al. CAR T cell-induced cytokine release syndrome is mediated by macrophages and abated by IL-1 blockade. Nat. Med. 24, 731-738 (2018).

73. Ahmed, S. et al. Haemophagocytic lymphohistiocytosis $(\mathrm{HLH})$ in patients with large B-cell lymphoma treated with standard of care (SOC) axicabtagene ciloleucel (Axi-cel). J. Clin. Oncol. 38 (suppl. 15), 8057 (2020).

74. McGonagle, D. \& McDermott, M. F. A proposed classification of the immunological diseases. PLoS Med 3, e297 (2006)

75. Grateau, G. et al. How should we approach classification of autoinflammatory diseases? Nat. Rev. Rheumatol. 9, 624 (2013).

76. Lehmberg, K. et al. Malignancy-associated haemophagocytic lymphohistiocytosis in children and adolescents. Br. J. Haematol. 170, 539-549 (2015).

77. Borgia, R. E., Gerstein, M., Levy, D. M., Silverman, E. D \& Hiraki, L. T. Features, treatment, and outcomes of macrophage activation syndrome in childhood-onset systemic lupus erythematosus. Arthritis Rheumatol. 70, 616-624 (2018)

78. Daver, N. et al. A consensus review on malignancyassociated hemophagocytic lymphohistiocytosis in adults. Cancer 123, 3229-3240 (2017).

79. Shimada, K., Kinoshita, T., Naoe, T. \& Nakamura, S. Presentation and management of intravascular large B-cell lymphoma. Lancet Oncol. 10, 895-902 (2009).

80. Vick, E. J., Patel, K., Prouet, P. \& Martin, M. G Proliferation through activation: hemophagocytic lymphohistiocytosis in hematologic malignancy. Blood Adv. 1, 779-791 (2017).

81. Chuang, H. C., Lay, J. D., Hsieh, W. C. \& Su, I. J. Pathogenesis and mechanism of disease progression from hemophagocytic lymphohistiocytosis to EpsteinBarr virus-associated T-cell lymphoma: nuclear factor-kB pathway as a potential therapeutic target. Cancer Sci. 98, 1281-1287 (2007).

82. Mănard, F. et al. Hodgkin lymphoma-associated hemophagocytic syndrome: a disorder strongly correlated with Epstein-Barr virus. Clin. Infect. Dis. 47, 531-534 (2008)

83. Sandler, R. D. et al. Diagnosis and management of secondary HLH/MAS following HSCT and CAR-T cell therapy in adults; a review of the literature and a survey of practice within EBMT centres on behalf of the autoimmune diseases working party (ADWP) and transplant complications working party (TCWP) Front. Immunol. 11, 524 (2020).

84. Sandler, R. D. et al. Haemophagocytic lymphohistiocytosis (HLH) following allogeneic haematopoietic stem cell transplantation (HSCT) time to reappraise with modern diagnostic and treatment strategies? Bone Marrow Transplant. 55 307-316 (2019)

85. Strippoli, R. et al. 2012. Amplification of the response to Toll-like receptor ligands by prolonged exposure to interleukin- 6 in mice: implication for the pathogenesis of macrophage activation syndrome. Arthritis Rheum. 64, 1680-1688 (2012)

86. Teachey, D. T. et al. Cytokine release syndrome after blinatumomab treatment related to abnormal macrophage activation and ameliorated with cytokinedirected therapy. Blood 121, 5154-5157 (2013)

87. Ceschi, A., Noseda, R., Palin, K. \& Verhamme, K. Immune checkpoint inhibitor-related cytokine release syndrome: analysis of WHO global pharmacovigilance database. Front. Pharmacol. 11, 557-557 (2020).

88. McGonagle, D., Sharif, K., O'Regan, A. \& Bridgewood, C. Interleukin-6 use in COVID-19 pneumonia related macrophage activation syndrome. Autoimmun. Rev. 19, 102537 (2020).

89. Bracaglia, C. et al. Elevated circulating levels of interferon- $\gamma$ and interferon- $\gamma$-induced chemokines characterise patients with macrophage activation syndrome complicating systemic juvenile idiopathic arthritis. Ann. Rheum. Dis. 76, 166-172 (2017).
90. Maude, S. L. et al. Tisagenlecleucel in children and young adults with B-cell lymphoblastic leukemia. N. Engl. J. Med. 378, 439-448 (2018).

91. Seder, R. A., Gazzinelli, R., Sher, A. \& Paul, W. E. Interleukin 12 acts directly on CD4+ T cells to enhance priming for interferon gamma production and diminishes interleukin 4 inhibition of such priming. Proc. Natl Acad. Sci. USA 90, 10188-10192 (1993).

92. Locatelli, F. et al. Emapalumab in children with primary hemophagocytic lymphohistiocytosis. N. Engl. J. Med. 382, 1811-1822 (2020)

93. De Benedetti, F. et al. OP0204 EMAPALUMAB, an interferon gamma (IFN-Y)-BLOCKING monoclonal antibody, in patients with macrophage activation syndrome (MAS) complicating systemic juvenile idiopathic arthritis (SJIA). Ann. Rheum. Dis. 78, 178 (2019).

94. Burn, T. N. et al. Genetic deficiency of interferon- $\gamma$ reveals interferon- $\gamma$-independent manifestations of murine hemophagocytic lymphohistiocytosis. Arthritis Rheumatol. 72, 335-347 (2020).

95. Gabay, C. et al. Open-label, multicentre, doseescalating phase II clinical trial on the safety and efficacy of tadekinig alfa (IL-18BP) in adult-onset Still's disease. Ann. Rheum. Dis. 77, 840-847 (2018).

96. Dinarello, C., Novick, D., Kim, S. \& Kaplanski, G. Interleukin-18 and IL-18 binding protein. Front. Immunol. 4, 289 (2013).

97. Shimizu, M. et al. Interleukin-18 for predicting the development of macrophage activation syndrome in systemic juvenile idiopathic arthritis. Clin. Immunol. 160, 277-281 (2015).

98. Weiss, E. S. et al. Interleukin-18 diagnostically distinguishes and pathogenically promotes human and murine macrophage activation syndrome. Blood 131, 1442-1455 (2018).

99. Inoue, N. et al. Cytokine profile in adult-onset Still's disease: comparison with systemic juvenile idiopathic arthritis. Clin. Immunol. 169, 8-13 (2016).

100. Romberg, N. et al. Mutation of NLRC4 causes a syndrome of enterocolitis and autoinflammation. Nat. Genet. 46, 1135-1139 (2014).

101. Calabrese, L. H. \& Calabrese, C. Cytokine release syndrome and the prospects for immunotherapy with COVID-19. Part 2: the role of interleukin 1. Cleve. Clin J. Med. https://doi.org/10.3949/ccjm.87a.ccc044 (2020).

102. Cavalli, G. et al. Interleukin-1 blockade with high-dose anakinra in patients with COVID-19, acute respiratory distress syndrome, and hyperinflammation: a retrospective cohort study. Lancet Rheumatol. 2 , e325-e331 (2020).

103. Pontali, E. et al. Safety and efficacy of early high-dose IV anakinra in severe COVID-19 lung disease. J. Allergy Clin. Immunol. 146, 213-215 (2020).

104. Sönmez, H. E., Demir, S., Bilginer, Y. \& Özen, S. Anakinra treatment in macrophage activation syndrome: a single center experience and systemic review of literature. Clin. Rheumatol. 37, 3329-3335 (2018).

105. La Rosée, P. et al. Recommendations for the management of hemophagocytic lymphohistiocytosis in adults. Blood 133, 2465-2477 (2019).

106. Cron, R. Q., Behrens, E. M., Shakoory, B., Ramanan, A. V. \& Chatham, W. W. Does vira hemorrhagic fever represent reactive hemophagocytic syndrome? J. Rheumatol. 42, 1078-1080 (2015).

107. Grom, A. A et al. Rate and clinical presentation of macrophage activation syndrome in patients with systemic juvenile idiopathic arthritis treated with canakinumab. Arthritis Rheumatol. 68, 218-228 (2016).

108. Hunter, C., Chizzonite, R. \& Remington, J. IL-1 beta is required for IL-12 to induce production of IFN-gamma by NK cells. A role for IL-1 beta in the T cellindependent mechanism of resistance against intracellular pathogens. J. Immunol. 155 4347-4354 (1995).

109. Micallef, M. J. et al. Interferon- $\gamma$-inducing factor enhances T helper 1 cytokine production by stimulated human T cells: synergism with interleukin-12 for interferon- $\gamma$ production. Eur. J. Immunol. 26, 1647-1651 (1996).

110. Frigault, M. J. et al. Tocilizumab not associated with increased infection risk after CAR T-cell therapy: implications for COVID-19? Blood 136, 137-139 (2020).

111. De Benedetti, F. et al. Randomized trial of tocilizumab in systemic juvenile idiopathic arthritis. N. Engl. J. Med. 367, 2385-2395 (2012)

112. Kobayashi, M., Takahashi, Y., Yamashita, H., Kaneko, H. $\Sigma$ Mimori, A. Benefit and a possible risk of tocilizumab 
therapy for adult-onset Still's disease accompanied by macrophage-activation syndrome. Mod. Rheumatol. 21, 92-96 (2011).

113. Yokota, S. et al. Macrophage activation syndrome in patients with systemic juvenile idiopathic arthritis under treatment with tocilizumab. J. Rheumatol. 42 712-722 (2015)

114. Norelli, M. et al. Monocyte-derived IL- 1 and IL- 6 are differentially required for cytokine-release syndrome and neurotoxicity due to CAR T cells. Nat. Med. $\mathbf{2 4}$, 739-748 (2018).

115. Ahmed, A. et al. Ruxolitinib in adult patients with secondary haemophagocytic lymphohistiocytosis: an open-label, single-centre, pilot trial. Lancet Haematol. 6, e630-e637 (2019)

116. Kacar, M. et al. Mixed results with baricitinib in biological-resistant adult-onset Still's disease and undifferentiated systemic autoinflammatory disease. RMD Open 6, e001246 (2020).

117. Hu, Q. et al. Tofacitinib in refractory adult-onset Still's disease: 14 cases from a single centre in China. Ann. Rheum. Dis. 79, 842-844 (2020).

118. Mehta, P. et al. COVID-19: consider cytokine storm syndromes and immunosuppression. Lancet 395 1033 (2020).

119. Ruscitti, P. et al. Lung involvement in macrophage activation syndrome and severe COVID-19: results from a cross-sectional study to assess clinical laboratory and artificial intelligence-radiological differences. Ann. Rheum. Dis. 79, 1152-1155 (2020).

120. McGonagle, D., Sharif, K., O'Regan, A. \& Bridgewood, C. Interleukin-6 use in COVID-19 pneumonia related macrophage activation syndrome. Autoimmun. Rev. 19, 102537 (2020)

121. Fardet, L. et al. Development and validation of the HScore, a score for the diagnosis of reactive hemophagocytic syndrome. Arthritis Rheumatol. 66, 2613-2620 (2014)
122. Sinha, P., Matthay, M. A. \& Calfee, C. S. Is a "Cytokine Storm" relevant to COVID-19? JAMA Intern. Med. 180, 1152-1154 (2020).

123. Chen, N. et al. Epidemiological and clinical characteristics of 99 cases of 2019 novel coronavirus pneumonia in Wuhan, China: a descriptive study. Lancet 395, 507-513 (2020).

124. Huang, C. et al. Clinical features of patients infected with 2019 novel coronavirus in Wuhan, China. Lancet 395, 497-506 (2020).

125. McGonagle, D., O'Donnell, J. S., Sharif, K., Emery, P. $\&$ Bridgewood, C. Immune mechanisms of pulmonary intravascular coagulopathy in COVID-19 pneumonia. Lancet Rheumatol. 2, e437-e445 (2020).

126. McGonagle, D., Plein, S., O’Donnell, J. S., Sharif, K. \& Bridgewood, C. Increased cardiovascular mortality in African Americans with COVID-19. Lancet Respir. Med. 8, 649-651 (2020).

127. Horby, P. et al. Dexamethasone in hospitalized patients with Covid-19 - preliminary report. N. Engl. J. Med. https://doi.org/10.1056/NEJMoa2021436 (2020).

128. Schulert, G. S. et al. Whole-exome sequencing reveals mutations in genes linked to hemophagocytic lymphohistiocytosis and macrophage activation syndrome in fatal cases of $\mathrm{H} 1 \mathrm{~N} 1$ influenza. J. Infect. Dis. 213, 1180-1188 (2016).

129. Hadjadj, J. et al. Impaired type I interferon activity and inflammatory responses in severe COVID-19 patients. Science 369, 718-724 (2020).

130. van der Made, C. I. et al. Presence of genetic variants among young men with severe COVID-19. JAMA 324 $1-11(2020)$

131. Blair, R. V. et al. ARDS and cytokine storm in SARS-CoV-2 infected Caribbean Vervets. Preprint at bioRxiv https://doi.org/10.1101/2020.06.18.157933 (2020).

132. Jiang, L. et al. COVID-19 and multisystem inflammatory syndrome in children and adolescents. Lancet Infect. Dis. 20, e276-e288 (2020).
133. Davies, P. et al. Intensive care admissions of children with paediatric inflammatory multisystem syndrome temporally associated with SARS-CoV-2 (PIMS-TS) in the UK: a multicentre observational study. Lancet Child Adolesc. Health 4, 669-677 (2020).

134. Levin, M. Childhood multisystem inflammatory syndrome - a new challenge in the pandemic. N. Engl. J.Med. 383, 393-395 (2020).

135. McCrindle, B. W. \& Manlhiot, C. SARS-CoV-2-related inflammatory multisystem syndrome in children: different or shared etiology and pathophysiology as Kawasaki disease? JAMA 324, 246-248 (2020).

136. Natoli, V., Rosina, S. \& Ravelli, A. Is macrophage activation syndrome in Kawasaki disease underrecognized? J. Rheumatol. https://doi.org/ 10.3899/jrheum.200361 (2020).

\section{Acknowledgements}

The authors thank A. Goenka for helpful comments.

Author contributions

All authors researched data for the article and made a substantial contribution to discussion of the content, writing and review/editing of the manuscript before submission.

\section{Competing interests}

The authors declare no competing interests.

Peer review information

Nature Reviews Rheumatology thanks R.O. Cron, A. Grom, L. Quartuccio and J. Pachlopnik-Schmidt for their contribution to the peer review of this work.

\section{Publisher's note}

Springer Nature remains neutral with regard to jurisdictional claims in published maps and institutional affiliations.

(c) Springer Nature Limited 2021 